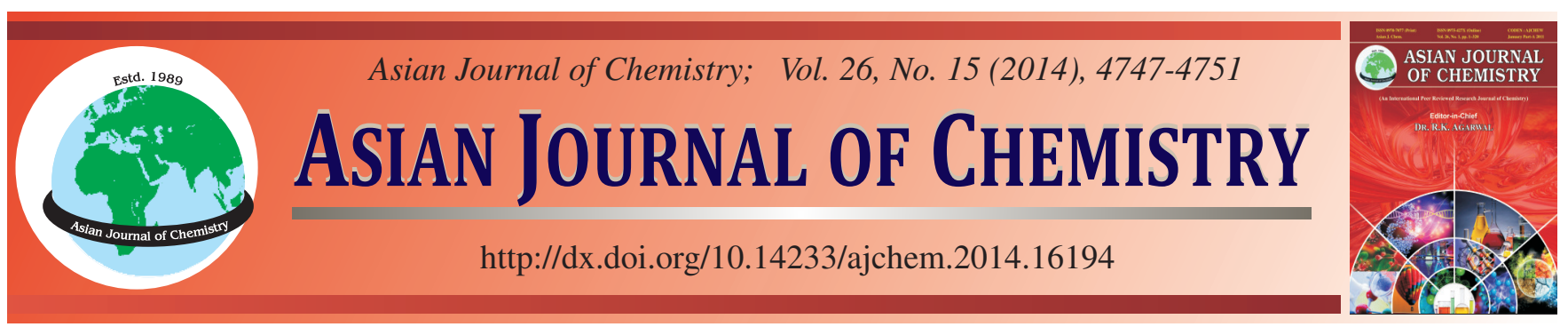

\title{
Amino Acid Promoted Ullmann Type Reaction with Low Catalyst Loading via Microwave Technology
}

\author{
K. Uma Maheshwar Reddy, K. Santosh Kumar and A. Panasa Reddy*
}

Department of Chemistry, University College of Engineering, Osmania University, Hyderabad-500 007, India

*Corresponding author: Tel: +91 40 27152892; Fax: +91 40 27095171; E-mail: reddypanasa@ gmail.com

Received: 16 August 2013;

Accepted: 23 December 2013;

Published online: 16 July 2014;

AJC-15564

\begin{abstract}
This work describes $\mathrm{CuBr}$-catalyzed cross-coupling of sterically hindered phenols with substituted aryl halides and hetero aryl halides under microwave conditions using commercially available amino acids as the reaction promoters and $\mathrm{K}_{3} \mathrm{PO}_{4}$ as the base. This protocol shown to tolerate sensitive functional groups like $\mathrm{CN}$, CHO etc., Especially, in case of 3-bromopyridine coupling with substituted phenols supported this letter in track record of the reactivity.
\end{abstract}

Keywords: Aryl halide, Phenol, Ligand, Microwave.

\section{INTRODUCTION}

Formation of C-O bond is a key step in synthetic chemistry due to the importance of diaryl ether unit represents a very important class of organic compounds ${ }^{1,2}$. The versatile strategies to access diaryl ethers directly by the reaction of aryl halide with phenols promoted by $\mathrm{Cu}$-reagents ${ }^{3}$. Ullmann discovered copper-catalyzed $O$-arylations of phenols with aryl halides ${ }^{4}$. However, synthetic scope of this reaction conditions are rather limited due to harsh reaction conditions, usually at high temperatures $\left(125-250{ }^{\circ} \mathrm{C}\right)$ in pyridine solvent, greater quantities of the copper catalyst required and the low to moderate yields. In recent modifications reported that the use of catalyst-ligand combinations enhance the reaction rate and allow the couplings to be carried out under milder reaction conditions in the presence of catalytic amount of copper ${ }^{5-7}$. Although several ligands such as 2,2,6,6,-tetramethyl-heptane3,5-dione (THMD) ${ }^{7 \mathrm{~b}}, \mathrm{~N}, \mathrm{~N}$-dimethylglycine ${ }^{8}$ have been reported as reaction promoters, with different $\mathrm{Cu}$ source and ligand combinations ${ }^{9,10}$ in the presence of different bases like $\mathrm{K}_{2} \mathrm{CO}_{3}$, $\mathrm{Cs}_{2} \mathrm{CO}_{3}$ in polar, non polar solvents, these catalytic systems still suffer from several common problems like stachiometric quantity of ligand loading and high temperature.

Yet, the development of this copper catalyzed Ullmanntype reaction is required all the times most catalytic systems still needed long reaction time (longer than $24 \mathrm{~h}$ ), relatively high temperature $\left(\geq 120^{\circ} \mathrm{C}\right)$ and high ratios of catalyst loading. We focused on development of cost effective, efficient catalytic system and the lowering the reaction time, temperature by adopting microwave technology ${ }^{11}$.

\section{EXPERIMENTAL}

General procedure: $\mathrm{CuBr}(2 \mathrm{~mol} \%)$, L-proline ( $5 \mathrm{~mol} \%)$, aryl halide (1 eq), $\mathrm{ArOH}(1.05 \mathrm{eq})$ and $\mathrm{K}_{3} \mathrm{PO}_{4}(2.5 \mathrm{eq})$ were suspended in DMF (3 mL) in a microwave vial. The tube was then evacuated and backfilled with nitrogen. The vial was sealed and placed in the Dicover-CEM microwave synthesizer; (temperature was controlled using the in-built calibrated IR sensor) and reaction mixture was irradiated at $70^{\circ} \mathrm{C}$ in microwave for 15-30 $\mathrm{min}$ (time variable for the different reactant). In 2-3 min reaction temperature attained to $70{ }^{\circ} \mathrm{C}$ and 3 bar pressure. The reaction mixture was cooled to room temperature and further diluted with water $(10 \mathrm{~mL})$ and ethyl acetate $(30 \mathrm{~mL})$. The organic layer was separated and back extracted twice with ethyl acetate $(20 \mathrm{~mL})$. The combined organic layers were dried over anhyd. $\mathrm{Na}_{2} \mathrm{SO}_{4}$ and concentrated via rota evaporator. The residue was purified through silica gel (60-120 mesh) column chromatography to afford diaryl ether product Scheme-I.

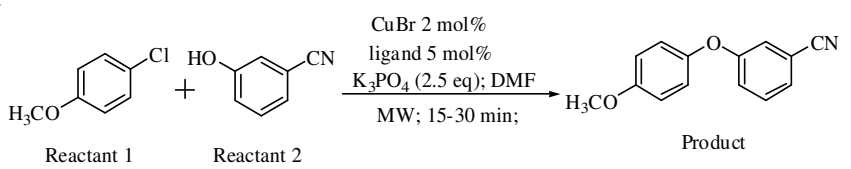

Scheme 1: Copper-catalyzed coupling of 4-chloroanisole with 3-hydroxy benzonitrile

3-(4-Methoxyphenoxy)benzonitrile (1c): Pale white colour solid, m.p. 106-108 ${ }^{\circ} \mathrm{C} ;{ }^{1} \mathrm{H}$ NMR (400 MHz, DMSOd 6 : $\delta(\mathrm{ppm}) 7.38(\mathrm{t}, J=8.0 \mathrm{~Hz}, 1 \mathrm{H}), 7.30(\mathrm{~d}, J=7.6 \mathrm{~Hz}, 1 \mathrm{H})$, 7.19-7.13 (m, 2H), 7.00-6.92 (m, 4H), 3.82 (s, 3H); ${ }^{13} \mathrm{C}$ NMR 
$\left(100 \mathrm{MHz}_{\mathrm{CDCl}}\right): \delta(\mathrm{ppm}) 159.2,156.8,148.4,130.5,125.7$, 121.7, 121.4, 119.9, 118.3, 115.2, 113.4, 55.6; MS (ESI) $\mathrm{m} / \mathrm{z}$ $(\mathrm{M}+\mathrm{H})^{+}$: 226.0; Anal. calcd. for $\mathrm{C}_{14} \mathrm{H}_{11} \mathrm{NO}_{2}$ : C, 74.65; $\mathrm{H}$, 4.92; N, 6.22; O, 14.21. Found: C, 74.75; H, 5.28.

3-(4-Methoxyphenoxy)benzaldehyde (2c): Colour less syrup, ${ }^{1} \mathrm{H}$ NMR (400 MHz, DMSO $\left.d_{6}\right): \delta(\mathrm{ppm}) 9.95$ (s, 1H), 7.63-7.59 (m, 2H), 7.32-7.30 (m, 2H), 7.08 (d, $J=6.8,2 \mathrm{H})$, $7.01(\mathrm{~d}, J=6.8,2 \mathrm{H}), 3.77(\mathrm{~s}, 3 \mathrm{H}) ;{ }^{13} \mathrm{C} \mathrm{NMR}\left(100 \mathrm{MHz}, \mathrm{CDCl}_{3}\right)$ : $\delta$ (ppm) 191.7, 159.2, 156.5, 149.1, 130.3, 124.1, 123.5, 121.2, 119.5, 116.8, 115.1, 55.6; MS (ESI) $\mathrm{m} / \mathrm{z}(\mathrm{M}+\mathrm{H})$ : 229.04; Anal. calcd. for $\mathrm{C}_{14} \mathrm{H}_{12} \mathrm{O}_{3}: \mathrm{C}, 73.67 ; \mathrm{H}, 5.30 ; \mathrm{O}, 21.03$. Found: C, 73.73; H, 5.41; Anal. calcd. for $\mathrm{C}_{14} \mathrm{H}_{12} \mathrm{O}_{3}$ : C, 73.67; H, 5.30; O, 21.03. Found: C, 73.72; H, 5.40.

4-(4-Methoxyphenoxy)benzaldehyde (3c): Pale white colour solid; m.p. 57-62 ${ }^{\circ} \mathrm{C}$; ${ }^{1} \mathrm{H}$ NMR (400 MHz, DMSO $d_{6}$ ): $\delta(\mathrm{ppm}) 9.90(\mathrm{~s}, 1 \mathrm{H}), 7.89(\mathrm{~d}, J=6.8 \mathrm{~Hz}, 2 \mathrm{H}), 7.11(\mathrm{~d}, J=6.8$ $\mathrm{Hz}, 2 \mathrm{H}), 7.06-7.02$ (m, 4H), 3.78 (s, 3H) ${ }^{13} \mathrm{C} \mathrm{NMR}(100 \mathrm{MHz}$, $\left.\mathrm{CDCl}_{3}\right): \delta(\mathrm{ppm}) 190.7,164.1,156.9,148.2,131.9,130.9$, 121.8, 116.7, 115.1, 55.6; MS (ESI) $\mathrm{m} / \mathrm{z}(\mathrm{M}+\mathrm{H})^{+}$: 229.04; Anal. calcd. for $\mathrm{C}_{14} \mathrm{H}_{12} \mathrm{O}_{3}: \mathrm{C}, 73.67 ; \mathrm{H}, 5.30 ; \mathrm{O}, 21.03$. Found: C, 73.73; H, 5.41.

2-(4-Methoxyphenoxy)-5-(benzyloxy)benzaldehyde (4c): Pale light colour solid, m.p. 121-125 ${ }^{\circ} \mathrm{C}$; ${ }^{1} \mathrm{H}$ NMR (400 MHz, DMSOd $\left.d_{6}\right): \delta(\mathrm{ppm}) 10.33(\mathrm{~s}, 1 \mathrm{H}), 7.47-7.31(\mathrm{~m}, 7 \mathrm{H})$, 7.05-6.89 (m, 5H), 5.15 (s, 2H), 3.75 (s, 3H); ${ }^{13} \mathrm{C} \mathrm{NMR} \mathrm{(100}$ $\left.\mathrm{MHz}, \mathrm{CDCl}_{3}\right): \delta$ (ppm) 189.2, 156.1, 155.0, 154.4, 150.6, 136.4, 128.6, 128.1, 127.6, 127.0, 124.3, 120.0, 119.9, 115.6, 111.0, 70.6, 55.7; MS (ESI) $m / z(\mathbf{M}+\mathrm{H})^{+}: 335.1$; Anal. calcd. for $\mathrm{C}_{21} \mathrm{H}_{18} \mathrm{O}_{4}$ : C, 75.43; H, 5.43; O, 19.14. Found: C, 75.52; H, 5.51 .

1-[4-(2-Methoxyphenoxy)phenyl]ethanone (5c): Colour less syrup, ${ }^{1} \mathrm{H}$ NMR $\left(400 \mathrm{MHz}, \mathrm{DMSO}_{6}\right): \delta(\mathrm{ppm}) 7.93(\mathrm{~d}, J$ $=9.6 \mathrm{~Hz} 2 \mathrm{H}), 7.31-7.15(\mathrm{~m}, 3 \mathrm{H}), 7.03(\mathrm{t}, J=7.6 \mathrm{~Hz} 1 \mathrm{H}), 6.88$ $(\mathrm{d}, J=9.6 \mathrm{~Hz}, 2 \mathrm{H}), 3.73$ (s, 3H), 2.51(s, 3H); ${ }^{13} \mathrm{C} \mathrm{NMR}(100$ $\mathrm{MHz}_{\mathrm{CDCl}}$ ): $\delta$ (ppm) 196.7, 162.4, 151.7, 143.2, 131.4, $130.4,126.1,122.4,121.3,115.7,113.0,55.8,26$ ppm. MS (ESI) $m / z(\mathrm{M}+\mathrm{H})^{+}:$243.05; Anal. calcd. for $\mathrm{C}_{15} \mathrm{H}_{14} \mathrm{O}_{3}$ : C, 74.36; H, 5.82; O, 19.81. Found: C, 74.45; H, 5.93.

3-(2-Formylphenoxy)benzonitrile (6c): Pale white colour solid, m.p. 80- $84{ }^{\circ} \mathrm{C}$; ${ }^{1} \mathrm{H}$ NMR (400 MHz, DMSO $\left.d_{6}\right)$ : $\delta(\mathrm{ppm}) 10.32(\mathrm{~s}, 1 \mathrm{H}), 7.91(\mathrm{~d}, J=1.2 \mathrm{~Hz}, 1 \mathrm{H}), 7.89-7.62(\mathrm{~m}$, $4 \mathrm{H}), 7.48(\mathrm{t}, J=1.2 \mathrm{~Hz}, 1 \mathrm{H}), 7.41(\mathrm{t}, J=7.6 \mathrm{~Hz}, 1 \mathrm{H}), 7.08(\mathrm{~d}$, $J=8.0 \mathrm{~Hz}, 1 \mathrm{H}) ;{ }^{13} \mathrm{C} \mathrm{NMR}\left(100 \mathrm{MHz}, \mathrm{CDCl}_{3}\right): \delta(\mathrm{ppm}) 189.4$, 158.1, 157.2, 136.9, 132.1, 129.5, 128.4, 127.4, 125.2, 124.4, 122.8, 120.1, 118.4, 113.3; MS (ESI) $\mathrm{m} / \mathrm{z}(\mathrm{M}+\mathrm{H})^{+}$: 224.03; Anal. calcd. for $\mathrm{C}_{14} \mathrm{H}_{9} \mathrm{NO}_{2}$ : C, 75.33; H, 4.06; N, 6.27; O, 14.33 . Found: C, 75.71; H, 4.12.

2-(O-tolyloxy)naphthalene (7c): Colourless syrup; ${ }^{1} \mathrm{H}$ NMR (400 MHz, DMSO $\left.d_{6}\right): \delta(p p m) 7.95(\mathrm{~d}, J=9.2 \mathrm{~Hz}, 1 \mathrm{H})$, $7.90(\mathrm{~d}, J=8.0 \mathrm{~Hz}, 1 \mathrm{H}), 7.76(\mathrm{~d}, J=8.0 \mathrm{~Hz}, 1 \mathrm{H}), 7.48-7.37$ $(\mathrm{m}, 3 \mathrm{H})$ 7.29-7.25 (m, 2H), 7.19-7.14 (m, 2H), 7.00 (d, $J=$ $8.0 \mathrm{~Hz}, 1 \mathrm{H}), 2.21(\mathrm{~s}, 3 \mathrm{H}) ;{ }^{13} \mathrm{C} \mathrm{NMR}\left(100 \mathrm{MHz}, \mathrm{CDCl}_{3}\right): \delta$ (ppm) 155.8, 154.3, 134.4, 131.5, 130.2, 129.8, 129.7, 127.7, 127.2, 126.4, 124.2, 120.1, 119.1, 111.6, 16.2; MS (ESI) $\mathrm{m} / \mathrm{z}$ $(\mathrm{M}+\mathrm{H})^{+}$: 235.01; Anal. calcd. for $\mathrm{C}_{17} \mathrm{H}_{14} \mathrm{O}: \mathrm{C}, \mathrm{C}, 87.15 ; \mathrm{H}$, 6.02; O, 6.83. Found: C, 87.23; H, 6.12.

5-(2-Isopropylphenoxy)pyridine-2-carbonitrile (8c): Pale yellow syrup, ${ }^{1} \mathrm{H}$ NMR $\left(400 \mathrm{MHz}, \mathrm{DMSO} d_{6}\right): \delta(\mathrm{ppm})$ $8.50(\mathrm{~d}, J=2.4 \mathrm{~Hz}, 1 \mathrm{H}), 8.01(\mathrm{~d}, J=8.0 \mathrm{~Hz}, 1 \mathrm{H}), 7.50(\mathrm{~d}, J=$
4.0, 1H), 7.37-7.31 (m, 3H), $7.10(\mathrm{dd}, J=3.4 \mathrm{~Hz}, 6.0 \mathrm{~Hz}, 1 \mathrm{H})$, 3.09-3.02 (m, 1H), $1.16(\mathrm{~d}, J=6.8 \mathrm{~Hz}, 6 \mathrm{H}) ;{ }^{13} \mathrm{C}$ NMR $(100$ $\left.\mathrm{MHz}, \mathrm{CDCl}_{3}\right): \delta$ (ppm) 157.5, 150.8, 141.3, 140.7, 129.5, 127.8, 127.6, 126.5, 122.5, 120.6, 117.2, 27.1, 23.02; MS (ESI) $m / z(\mathrm{M}+\mathrm{H})^{+}$: 239.02; Anal. calcd. for $\mathrm{C}_{15} \mathrm{H}_{14} \mathrm{~N}_{2} \mathrm{O}: \mathrm{C}, 75.61$; H, 5.92; N, 11.76; O, 6.71. Found: C, 75.74; H, 5.99.

4-(2-Isopropylphenoxy)isoquinoline (9c): Pale brown syrup, ${ }^{1} \mathrm{H}$ NMR (400 MHz, DMSO $\left.d_{6}\right): \delta(\mathrm{ppm}) 9.11(\mathrm{~s}, 1 \mathrm{H})$, $8.22(\mathrm{~d}, J=8.4 \mathrm{~Hz}, 1 \mathrm{H}), 8.16(\mathrm{~d}, J=8.4,1 \mathrm{H}), 7.90-7.77(\mathrm{~m}$, $3 \mathrm{H}), 7.48$ (q, 1H), 7.24-7.22 (m, 2H) 6.92-6.90 (m, 1H), 3.28 $(\mathrm{m}, 1 \mathrm{H}), 1.25(\mathrm{~d}, J=7.2 \mathrm{~Hz}, 6 \mathrm{H}) ;{ }^{13} \mathrm{C} \mathrm{NMR}\left(100 \mathrm{MHz}, \mathrm{CDCl}_{3}\right)$ : $\delta$ (ppm) 153.4, 149.5, 146.7, 139.7, 130.1, 129.5, 128.9, 127.8, 127.2, 127.2, 127.1, 127.7, 121.1, 119.3, 27.3, 23.0; MS (ESI) $m / z(\mathrm{M}+\mathrm{H})^{+}: 264.01 ; \mathrm{C}_{18} \mathrm{H}_{17} \mathrm{NO}$ Anal. calcd. for $\mathrm{C}_{15} \mathrm{H}_{14} \mathrm{~N}_{2} \mathrm{O}$ : C, 82.10; H, 6.51; N, 5.32; O, 6.08. Found: C, 82.24; H, 6.45.

2-(Pyridin-3-yloxy)benzaldehyde (10c): Yellow crystalline solid; m.p. 75-79 ${ }^{\circ} \mathrm{C} ;{ }^{1} \mathrm{H}$ NMR $(400 \mathrm{MHz}$, $\left.\mathrm{DMSO}_{6}\right): \delta(\mathrm{ppm}) 10.41(\mathrm{~s}, 1 \mathrm{H}), 8.50(\mathrm{~d}, J=2.8 \mathrm{~Hz}, 1 \mathrm{H})$, 8.45 (dd, $J=1.2 \mathrm{~Hz}, 4.4 \mathrm{~Hz}, 1 \mathrm{H}), 7.89$ (dd, $J=2.0 \mathrm{~Hz}, 8.0 \mathrm{~Hz}$, 1H), $7.70(\mathrm{~d}, J=7.6 \mathrm{~Hz}, 1 \mathrm{H}), 7.60-7.57(\mathrm{~m}, 1 \mathrm{H}), 7.50-7.47$ $(\mathrm{m}, 1 \mathrm{H}), 7.36(\mathrm{t}, J=7.6 \mathrm{~Hz}, 1 \mathrm{H}), 7.03(\mathrm{~d}, J=8.4 \mathrm{~Hz}, 1 \mathrm{H}) ;{ }^{13} \mathrm{C}$ NMR (100 MHz, $\mathrm{CDCl}_{3}$ ): $\delta$ (ppm) 186.6, 158.8, 153.1, 145.3, $141.5,135.9,129.0,127.1,126.3,124.4,124.3,118.4$; MS (ESI) $m / z(\mathrm{M}+\mathrm{H})^{+}: 200.2$; Anal. calcd. for $\mathrm{C}_{12} \mathrm{H}_{9} \mathrm{NO}_{2}$ : C, 72.35; H, 4.55; N, 7.03; O, 16.06. Found: C, 72.45; H, 4.64.

4-Methoxy-3-(pyridin-3-yloxy)benzaldehyde (11c): Pale yellow syrup, ${ }^{1} \mathrm{H}$ NMR (400 MHz, DMSO $\left.d_{6}\right): \delta(\mathrm{ppm})$ $9.84(\mathrm{~s}, 1 \mathrm{H}), 8.35(\mathrm{~s}, 2 \mathrm{H}), 7.72(\mathrm{dd}, J=2.0,8.4 \mathrm{~Hz}, 1 \mathrm{H}), 7.52$ $(\mathrm{d}, J=1.6 \mathrm{~Hz}, 1 \mathrm{H}), 7.25-7.32(\mathrm{~m}, 2 \mathrm{H}), 7.12(\mathrm{~d}, J=8.4 \mathrm{~Hz}$, 1H), 3.92 (s, 3H): ${ }^{13} \mathrm{C}$ NMR (100 MHz, $\left.\mathrm{CDCl}_{3}\right): \delta(\mathrm{ppm}) 190.0$, 156.3, 153.7, 144.9, 144.3, 140.1, 130.3, 129.0, 124.2, 120.5, 112.3, 56.2 ppm; MS (ESI) m/z (M + H $)^{+}$: 230.1; Anal. calcd. for $\mathrm{C}_{13} \mathrm{H}_{11} \mathrm{NO}_{3}$ : C, 68.11; H, 4.84; N, 6.11; O, 20.94. Found: C, 68.23; H, 4.95.

1-(4-(Thiazol-2-yloxy)phenyl)ethanone (12c): Pale brown semisolid; ${ }^{1} \mathrm{H}$ NMR (400 MHz, DMSO $\left.d_{6}\right): \delta(\mathrm{ppm})$ $8.06(\mathrm{~d}, J=6.8 \mathrm{~Hz}, 2 \mathrm{H}), 7.46(\mathrm{~d}, J=6.8 \mathrm{~Hz}, 2 \mathrm{H}), 7.35$ (d, $J=$ $6.4 \mathrm{~Hz}, 2 \mathrm{H}), 2.60(\mathrm{~s}, 3 \mathrm{H}) ;{ }^{13} \mathrm{C} \mathrm{NMR}\left(100 \mathrm{MHz}, \mathrm{CDCl}_{3}\right): \delta$ (ppm) 196.6, 172.0, 158.9, 137.6, 134.2, 130.4, 119.4, 113.9, $26.5 \mathrm{ppm}$. MS (ESI) $\mathrm{m} / \mathrm{z}(\mathrm{M}+\mathrm{H})^{+}$: 220; Anal. calcd. for $\mathrm{C}_{11} \mathrm{H}_{9} \mathrm{NO}_{2} \mathrm{~S}$ : C, 60.26; H, 4.14; N, 6.39; O, 14.59; S, 14.62 . Found: C, 60.35; H,4.22.

6-(Pyridin-3-yloxy)pyridine-3-carbonitrile (13c): Pale yellow gummy solid; ${ }^{1} \mathrm{H}$ NMR $\left(400 \mathrm{MHz}, \mathrm{DMSO} d_{6}\right): \delta(\mathrm{ppm})$ $8.66(\mathrm{~d}, J=2.4 \mathrm{~Hz}, 1 \mathrm{H}), 8.53-8.50(\mathrm{~m}, 2 \mathrm{H}), 8.38$ (d, $J=8.8$ $\mathrm{Hz}, 1 \mathrm{H}), 7.74$ (d, $J=8.4 \mathrm{~Hz}, 1 \mathrm{H}), 7.54-7.51$ (m, 1H), 7.37 (d, $J=8.8 \mathrm{~Hz}, 1 \mathrm{H}) .{ }^{13} \mathrm{C}$ NMR $\left(100 \mathrm{MHz}, \mathrm{CDCl}_{3}\right): \delta(\mathrm{ppm}) 164.7$, 162.5, 151.7, 149.2, 146.7, 143.6, 142.5, 129.1, 127.1, 116.4, 112.3, 104.8. MS (ESI) $\mathrm{m} / z(\mathrm{M}+\mathrm{H})^{+}$: 198.2; Anal. calcd. for $\mathrm{C}_{11} \mathrm{H}_{7} \mathrm{~N}_{3} \mathrm{O}: \mathrm{C}, 67.00 ; \mathrm{H}, 3.58 ; \mathrm{N}, 21.31 ; \mathrm{O}, 8.11$. Found: $\mathrm{C}$, 67.23; H, 3.95 .

5-(Quinolin-3-yloxy)pyridine-3-carbaldehyde(14c): pale yellow syrup, ${ }^{1} \mathrm{H}$ NMR $\left(400 \mathrm{MHz}, \mathrm{DMSO} d_{6}\right): \delta(\mathrm{ppm})$ 10.12 (s, 1H), $8.93(\mathrm{~d}, J=1.6 \mathrm{~Hz}, 1 \mathrm{H}), 8.89$ (dd, $J=1.6 \mathrm{~Hz}$, $4.0 \mathrm{~Hz}, 1 \mathrm{H}), 8.81(\mathrm{~d}, J=2.8 \mathrm{~Hz}, 1 \mathrm{H}), 8.32(\mathrm{dd}, J=1.2 \mathrm{~Hz}, J=$ $8.4 \mathrm{~Hz}, 1 \mathrm{H}), 8.12(\mathrm{~d}, J=8.4 \mathrm{~Hz}, 1 \mathrm{H}), 7.88-7.87(\mathrm{~m}, 1 \mathrm{H}), 7.67$ $(\mathrm{d}, J=2.8 \mathrm{~Hz}, 1 \mathrm{H}), 7.64(\mathrm{~s}, 1 \mathrm{H}), 7.57-7.59(\mathrm{~m}, 1 \mathrm{H}) ;{ }^{13} \mathrm{C} \mathrm{NMR}$ $\left(100 \mathrm{MHz}, \mathrm{CDCl}_{3}\right): \delta$ (ppm) 191.9, 153.5, 153.3, 149.8, 146.3, 146.1, 145.0, 135.4, 132.2, 131.5, 128.7, 124.3, 122.9, 122.0, 
114.5; MS (ESI) $m / z(\mathrm{M}+\mathrm{H})^{+}$: 251.5; Anal. calcd. for $\mathrm{C}_{15} \mathrm{H}_{10} \mathrm{~N}_{2} \mathrm{O}_{2}$ : C, 71.99; H, 4.03; N, 11.19; O, 12.79 Found: C, $72.08 ; \mathrm{H}, 4.14$.

\section{RESULTS AND DISCUSSION}

In preliminary studies, reaction of 4-chloroanisole with 3-hydroxy benzonitrile in DMF at $110{ }^{\circ} \mathrm{C}$ in the presence of $\mathrm{K}_{3} \mathrm{PO}_{4}$ using $5 \mathrm{~mol} \%$ of $\mathrm{CuBr}$ without using additives the reaction was examined subsequent conventional heating and microwave reaction. Conventional heating resulted $55 \%$ of product selectivity in $22 \mathrm{~h}$ at $110^{\circ} \mathrm{C}$, where $72 \%$ product selectivity was detected under microwave heating in $20 \mathrm{~min}$. By comparison, reaction completed in $15 \mathrm{~min}$ in the presence of $10 \mathrm{~mol} \%$ of L-proline under microwave heating (CEM synthesizer) and product selectivity also increased to $95 \%$. It has been suggested that such additives would increase the solubility of copper salts by preventing their aggregation and they could also enhance the reactivity. The reaction screened with using commercially available amino acids such as L-proline, $\mathrm{N}$-methyl proline, pyrrole-2-carboxilic acid and 2-picolinic acid, in DMF and $\mathrm{K}_{3} \mathrm{PO}_{4}$ as base and results are summarized in Table-1.

After screening of above four ligands, L-proline was found to be a superior ligand combination with $\mathrm{CuBr}$ to serve as better ligand-catalytic system to make diaryl ethers at lower temperature in very shorter reaction time under microwave conditions. After optimizing the reaction conditions, we subsequently extended the scope of Ullmann-type coupling reaction using $5 \mathrm{~mol} \%$ of L-proline without affecting the product selectivity. All the reactions performed efficiently using $2 \mathrm{~mol}$ $\%$ of $\mathrm{CuBr}, 5 \mathrm{~mol} \%$ of ligand under microwave conditions in $\mathrm{DMF}$ and $\mathrm{K}_{3} \mathrm{PO}_{4}$ as base at $70{ }^{\circ} \mathrm{C}$. To the best of our knowledge, most of the diaryl ether formation reactions reported with highly reactive aryl iodides with phenols, few reports have been found with less reactive aryl chloride and moderate reactive aryl bromides and very few reports on hetero aromatic halides. We report here, $\mathrm{CuBr}$ and L-proline is the best combination for the $\mathrm{C}-\mathrm{O}$ bond formation of less reactive aryl/heteroaryl chlorides and also moderate reactive aryl/hetero-aryl bromides with sterically hindered phenols and hetero-aryl alcohols with excellent yields using low catalyst-ligand loading under microwave irradiation. During recent years, microwaves have been extensively used for carrying out chemical reactions and have become a useful non-conventional energy source for performing organic synthesis ${ }^{12,13}$. This protocol mainly refer the ligand loading, solvent ratio, time, temperature and yields. And also observed stability in the presence of sensitive functional groups. The isolated yields compared with conventional heating and results are summarized in Table-2.
We were curious about effect of substituent on the rate of the coupling reaction. Generally, ortho-substituted phenol coupling partners are often challenging due to steric hindrance. By using the optimized conditions ortho-substituted halides could be coupled with a variety of phenols (entriy 6). Electrondonating groups on the phenol and electron-withdrawing groups on the aryl halides make the reaction favourable. Electron withdrawing group of ortho-substituted phenols effectively formed ethers with hetero-aryl halides (entries $7-11$ ) gave above $85 \%$ yields. The steric hindrance of phenols usually slightly disfavoured for the reaction. 4-Chloroacetophenone was used as the substrate and reacted with 2methoxyphenol resulted in $90 \%$, where considerably lower yield in conventional heating. Spectacular electronic effects were observed when 2-Isoproylphenol was submitted to coupling with het hetero-aryl chlorides (entries 8,9) more than $80 \%$ yield. Only slight decrease in the reaction rate was noted with electron rich and electron withdrawing substituted 2chloro-5-cyanopyridine (entry 9) here -CN group was well tolerated on the aryl chloride. We please to note that we could quantitatively couple 2-bromo napthalene with the sterically hindered ortho-cresol under these conditions (entry 7) with $91 \%$ yield.

Next, the cross-coupling reaction between hetero-aryl halides with substituted phenols (entries 8-12) and hetero-aryl alcohols were examined (entries 13 and 14). The one, we identified the substituted phenols coupling with 3-halopyridine case is much differential. Where the much difference observed in yield while comparing with the conventional and microwave technologies. The earlier literature, failed to provide coupling reaction of 3-halopyridines ${ }^{12}$ because less reactive than 2 halopyridine. This letter strongly, supported here in getting extreme reactivity observed in microwave condition (entries $10,11,14)$. The other substituted phenols possessing sensitive functional groups are well tolerated during coupling under microwave and also afforded reasonable yields (entries 1, 2, $8,11,13,14)$. Hetero-aryl halides such as 3-chloro pyridine (entries 10, 11), 3-Cyano-5-chloropyridines, 4-Chloroisoquinoline, 2-bromothiazole (entry 12), 5-bromonicotinaldehyde (entry 14) could be coupled with electro-deficient, electronneutral and hindered phenols. However, 5-membered ring hetero-aryl halides containing two hetero atoms such as 2-bromothiazole (entry 12) also gave product reaction with 3-hydroxypyridine in excellent yields under these reaction conditions. The $\mathrm{C}-\mathrm{O}$ bond formation of 3-hydroxyquinoline (entry 14) with a substituted pyridine also proceeded smoothly and also gave excellent yield $86 \%$. Common functional groups, including cyano, aldehydes and ketones were tolerated under the reaction conditions employed.

TABLE-1

COUPLING REACTION OF 4-CHLOROANISOL WITH PHENOL UNDER THE CATALYSIS OF CuBr AND AMINO ACIDS

\begin{tabular}{ccccc}
\hline Entry & Catalyst & Ligand & Conventional (Cal. Yield \%)* & MW (Cal. Yield \%)* \\
\hline 1 & $\mathrm{CuBr}$ & L-Proline & 61 & 95 \\
2 & $\mathrm{CuBr}$ & $N$-Methyl proline & 48 & 86 \\
3 & $\mathrm{CuBr}$ & 2-Picolinic acid & 52 & 82 \\
4 & $\mathrm{CuBr}$ & Pyrrole-2-carboxilic acid & 55 & 85 \\
5 & $\mathrm{CuI}$ & L-Proline & 53 & 81 \\
\hline
\end{tabular}

Reaction conditions: $\mathrm{CuBr}$ powder (2 mol \%), ligand (5 mol \%), 4-chloroanisole (1.0 eq), 3-hydroxy benzonitrile (1.05 eq), $\mathrm{K}_{3} \mathrm{PO}_{4}(2.5$ eq), DMF MW 15-30 min 
TABLE-2

CuBr-CATALYZED DIARYL ETHER FORMATION OF ARYL/HETERO-ARYL

HALIDES WITH SELECTED PHENOLS IN THE PRESENCE OF L-PROLINE

\begin{tabular}{|c|c|c|c|c|c|c|}
\hline Entry & $\operatorname{ArX}(\mathrm{a})$ & $\mathrm{ArOH}(\mathrm{b})$ & Product (c) & Temp $\left({ }^{\circ} \mathrm{C}\right)$ & Conventional yield (\%) & MW yield $(\%)$ \\
\hline 1 & & & & 70 & 57 & 95 \\
\hline 2 & & & & 70 & 59 & 93 \\
\hline 3 & & & & 70 & 61 & 90 \\
\hline 4 & & & & 70 & 48 & 86 \\
\hline 5 & & & & 75 & 67 & 90 \\
\hline 6 & & & & 70 & 46 & 87 \\
\hline 7 & & & & 70 & 59 & 91 \\
\hline 8 & & & & 80 & 55 & 87 \\
\hline 9 & & & & 90 & 47 & 88 \\
\hline 10 & & & & 90 & 63 & 90 \\
\hline 11 & & & & 90 & 49 & 86 \\
\hline 12 & & & & 80 & 51 & 91 \\
\hline 13 & & & & 70 & 55 & 90 \\
\hline 14 & & & & 90 & 38 & 86 \\
\hline
\end{tabular}

The best results were observed in microwave irradiation rather thermal heating with remarkable reduction in reaction time because of homogeneous heating throughout the reaction media by microwave irradiation as compared to convection currents in thermal heating. Evidently, the microwave irradiation increased the reaction rate and selectivity rather than conventional method taking much longer reaction time (requires 20$30 \mathrm{~h}$ at $\left.110-130^{\circ} \mathrm{C}\right)$.

\section{Conclusion}

We have successfully demonstrated economical and operationally simple $\mathrm{CuBr}$-L-proline catalytic synthesis of substituted di aryl, hetero-aryl ethers from the coupling of substituted phenols towards halo pyridines, haloquinolines and bromo benzothiazole in excellent yields with low loading ligands in shorter time under microwave conditions. The best results were observed in microwave irradiation rather than 
conventional heating with remarkable reduction in reaction time.

\section{ACKNOWLEDGEMENTS}

The authors acknowledged to Osmania University for providing the research facility and the direct contributions for the staff of Department of Chemistry and Analytical team.

\section{REFERENCES}

1. (a) J. Lindley, Tetrahedron, 40, 1433 (1984); (b) A.V.R. Rao, M.K Gurjar, K.L. Reddy and A.S. Rao, Chem. Rev., 95, 2135 (1995); (c) J. Scott Sawyer, Tetrahedron, 56, 5045 (2000); (d) C. Zhong, J. He, C. Xue and Y. Li, Bioorg. Med. Chem., 12, 4009 (2004).

2. (a) S.B. Singh and G.R. Pettit, J. Org. Chem., 55, 2797 (1990); (b) L. Liu, A. Siegmund, N. Xi, P. Kaplan-Lefko, K. Rex, A. Chen, J. Lin, J. Moriguchi, L. Berry, L. Huang, Y. Teffera, Y. Yang, Y. Zhang, S.F. Bellon, M. Lee, R. Shimanovich, A. Bak, C. Dominguez, M.H. Norman, J.-C. Harmange, I. Dussault and T.-S. Kim, J. Med. Chem., 51, 3688 (2008); (c) S. Caron, N.M. Do, J.E. Sieser, D.C. Whritenour and P.D. Hill, Org. Process Res. Dev., 13, 324 (2009); (d) F.F. Yong, Y.C. Teo, Y.K. Yan and G.L. Chua, Synlett, 101 (2012); (e) W.M. Draper and J.E. Casida, J. Agric. Food Chem., 31, 1201 (1983); (f) C. Tomlin, The Pesticide Manual; Corp Protection Publication: Farnham, UK, edn 10 (1994).
3. (a) J. Lindley, Tetrahedron, 40, 1433 (1984); (b) F. Theil, Angew. Chem. Int. Ed., 38, 2345 (1999); (c) F. Thomas, S. Schulz, H. Mansikkamäki and M. Nieger, Angew. Chem. Int. Ed., 42, 5641 (2003).

4. (a) F. Ullmann, Ber. Dtsch. Chem. Ges, 36, 2382 (1903); (b) F. Ullmann, Chem. Ber., 37, 853 (1904).

5. H.J. Cristau, P. Cellier, S. Hamada, J.F. Spindler and M. Taillefer, Org. Lett., 6, 913 (2004)

6. (a) A. Kiyomori, J.F. Marcoux and S.L. Buchwald, Tetrahedron Lett., 40, 2657 (1999); (b) T.D. Quach and R.A. Batey, Org. Lett., 5, 1381 (2003).

7. (a) R.K. Gujadhur, C.G. Bates and D. Venkataraman, Org. Lett., 3, 4315 (2001); (b) E. Buck, Z.J. Song, D. Tschaen, P.G. Dormer, R.P. Volante and P.J. Reider, Org. Lett., 4, 1623 (2002).

8. D. Ma and Q. Cai, Org. Lett., 5, 3799 (2003).

9. (a) P.Y.S. Lam, C.G. Clark, S. Saubern, J. Adams, M.P. Winters, D.M.T. Chan and A. Combs, Tetrahedron Lett., 39, 2941 (1998); (b) D.A. Evans, J.L. Katz and T.R. West, Tetrahedron Lett., 39, 2937 (1998).

10. J.-F. Marcoux, S. Doye and S.L. Buchwald, J. Am. Chem. Soc., 119, 10539 (1997).

11. (a) J.-F. Marcoux, S. Doye and S.L. Buchwald, J. Neurol. Neurosurg. Psychiatry, 55, 126 (1992).; (b) W. Deng, Y. Xu and Q.X. Guo, Chin. Chem. Lett., 16, 327 (2005).

12. R.N. Gedye and J.B. Wei, Can. J. Chem., 76, 525 (1998).

13. S. Noël, L. Djakovitch and C. Pinel, Tetrahedron Lett., 47, 3839 (2006). 